\title{
A FINE MIST OF VERY SMALL COMET DUST PARTICLES
}

\author{
J. Mayo Greenberg \\ Laboratory Astrophysics, Huygens Laboratory, Wassenaarseweg \\ 78, 2300 RA Leiden, Netherlands
}

\section{ABSTRACT}

A comet nucleus considered as an aggregate of interstellar dust would produce a mist of very finely divided (radius $\sim 0.01 \mu \mathrm{m}$ ) particles of carbon and metal oxides accompanying the larger dust grains. These small particles which are very abundant in the interstellar dust size spectrum would provide substantial physical effects because of their large surface area. They may show up strongly in particle detectors on the Halley probes. A strong basis for serfous consideration of these particles comes from the other evidence that interstellar dust grains are the building blocks of comets; e.g. (1) the explanation of the "missing" carbon in comets; (2) The $\mathrm{S}_{2}$ molecule detection which suggests that the comet solid ice materials have been previously subjected to ultraviolet radiation (as are Interstellar grains) before aggregation into the comet; (3) the predicted dust to gas ratio.

\section{INTRODUCTION}

Very little consideration has been given to the possibility of cometary particles as small as $0.01 \mu$, radius. However, if comets are aggregated interstellar dust there is good reason to expect that such particles should provide a very significant contribution to the cometary dust spectrum.

Theoretical and observational evidence is accumulating to support the hypothesis that comets formed and remained cold after aggregation. Theoretical studies by Biermann and Michel /1/ led to the conclusion that if comets aggregated in the presolar nebula disk they formed at a distance $\sim 10^{4} \mathrm{~A} . \mathrm{U}$. from the sun. More recently, Biermann and Mestel /2/ have provided reasons to expect that comets formed in a protocometary nebula within a cloud fragment gravitationally bound to the presolar nebula but one in which smaller bodies, like comets, are preferentially formed without a central condensation, as suggested some time earlier by Cameron /3/. In both of the above situations comets had to have formed directly out of the existing molecular cloud material which is (other than $H$ and $\mathrm{He}$ ) predominantly bound in interstellar dust.

Even if comets formed as near to the sun as the Uranus-Neptunus region, it has been shown by computer simulations that they must not have formed as a result of fragmentation of larger bodies but rather are in their original state $/ 4 /$.

It is plausible to assume, therefore, that the comets which occupy the oort cloud condenged in a cold medium and have remained very cold during the subsequent $4.510^{9}$ years. This leads to the comet being made up of aggregated interstellar dust whose temperature probably did not exceed $\sim 15 \mathrm{~K}$ so that at least most, if not all, of the volatile components would survive in their original form. Inevitably then the less volatile and refractory components are imbedded in a matrix of ices of which $\mathrm{H}_{2} \mathrm{O}$ is the largest single component $/ 5,6,7 /$. This model has been shown to provide a quantitative explanation of the "missing carbon" in comets $/ 8,6 /$, and the dust to gas ratio /6,7/. A'Hearn et al $/ 9 /$ have attributed the existence of $s_{2}$ in a comet spectrum to intersteilar dust irradiated before being incorporated into the comet nucleus.

VERY SMALL INTERSTELLAR DUST GRAINS $(a<0.01 \mu \mathrm{m})$

It has been recognized since the discovery of far ultraviolet interstellar extinction that this is produced by particles smaller than $0.1 \mu \mathrm{m}$. It has now been shown that a basic property of this extinction can only be explained if all the particles responsible for the wavelength dependent extinction shortward of $\lambda \approx 2500 \AA$ may be characterized as being of radii $<0.01 \mu \mathrm{m} / 10 /$. The conclusion is that, for every tenth micron sized grain there are 
perhaps a thousand hundredth micron sized grains. This result has been further supported in a comprehensive study of the FUV extinction by more than 100 stars deduced from IUE spectra for 142 stars /11/. Based on the amount of FUV extinction we predict that for every tenth micron sized interstellar grain there are a thousand hundredth micron (or less) sized grains of which about a half are composed of some form of carbon and a half are some silicate or metallic oxide composition. We thus have a bimodal distribution of very small metallic and dielectric grains.

VERY SMALL COMET DUST GRAINS $(\mathrm{a}<0.01 \mu \mathrm{m})$

No direct evidence for comet particles less than $0.01 \mu \mathrm{m}$ seems to exist. However, one should expect that as the volatiles of a comet dust particle, considered as an aggregate of interstellar dust particles, evaporate they should release a cloud of small carbon and silicate (or metallic oxide) grains which were trapped in the icy matrix. Indirect evidence is provided by detection of such particles in meteor streams /12/ which may be presumed to have originated in comets.

These particles are dynamically quite different from the more classical sized comet grains. Electromagnetic forces become important for very small charged particles/13/. As has been pointed out by Wallis and Hassan $/ 14 /$, In their calculations relevant to particles a bit larger than the ones we propose here, one expects $0.01 \mu m$ dielectric (silicate) grains to suffer little radiation pressure and they may be accelerated transverse to the Giotto spacecraft behind its shield and impact the detectors. Since the hundredth micron size particles are orders of magnitude more abundant than the larger particles, any detection device which is sensitive to particles of mass $\simeq 10^{-17} \mathrm{gm}$ would almost certainly be saturated. This is of great concern for many of the dust experiments (see e.g. /15/)

Another effect of the very small grains could be the role their large surface area plays in the chemistry of the coma. Also their strong coupling to the gas in combination with their mass ( 1/5 of the gas) could exert signiflcant influences on the tail.

There appear to be good reasons to give careful and detailed attention to the effects of exceedingly small particles on the chemical and dynamical evolution of the coma and tail of comets.

\section{ACKNOWLEDGEMENT}

I should like to thank Hugo Fechtig for suggesting that I look into the possibility of very small comet grains.

\section{REFERENCES}

1. L. Biermann, L.K.W. Michel, Moon \& Planets, 18, 447 (1978).

2. L. Biermann, Phil. Trans, R. Soc. London, A303, 351 (1981).

3. A.G.W. Cameron, Icarus, 18, 407 (1973).

4. R. Greenberg, Paper presented at Dynamics \& Origin of Comets, Rome (1984).

5. J.M. Greenberg, in Comets, ed. L. Wilkening (Univ. of Arizona Press), 131 (1982).

6. J.M. Greenberg, in Cometary Exploration, ed. T.J. Combosi, Hung. Acad. Sc1., 23 (1983)

7. J.M. Greenberg, in Asteroids, Comets, Meteors, ed. C.-I. Lagerkvist and H. Rickman, Uppsala University Press, 259 (1983).

8. A.H. Delsemme, in Comets, ed. L. Wilkening, Univ. of Arizona Press, 85 (1982).

9. M. A'Hearn, P. Feldman, in Proceedings of Workshop on Ices in the Solar System, Nice (1984).

10. J.M. Greenberg and G. Chlewicki, Astrophys. J., 272, 563 (1983).

11. G. Chlewicki, J.M. Greenberg, S. Aiello, B. Barsella, P. Patriarchi, M. Perionotto, in: Proceedings 4th European IUE Conference ESA SP-218, 507 (1984).

12. S.F. Singer \& J.E. Stanley, in Solid Particles in the Solar System, Reidel, 329 (1979).

13. T. Muka1, Astron. Astrophys. 99 1 (1981).

14. M.K. Walits and M.H.A. Hassan, in Cometary Exploration, ed. T.I. Combosi, Hung. Acad. Sci., 57, (1983).

15. H. Fechtig, in Cometary Exploration, ed. T.I. Combosi, Hung. Acad. Sc1., 57, 203. 